\title{
LA FLECHA DEL TIEMPO. UN ACERCAMIENTO INTERDISCIPLINAR DE LA FÍSICAA LA HISTORIA
}

\author{
THE ARROW OF TIME \\ AN INTERDISCIPLINARY APPROACH FROM PHYSICS TO HISTORY
}

\begin{abstract}
RESUMEN
Este artículo intenta un ejercicio de acercamiento disciplinar entre dos ciencias con distintos objetos del conocimiento que coinciden en el estudio del tiempo como coordenada fundamental. Esta noción paradójicamente hace la diferencia entre ambas, por ello será aquí abordada como factor de contrastación entre la concepción lineal que de ella prevalece en la Historia, versus la multidimensionalidad desde la que se le piensa en la Física. Los extraordinarios avances experimentados en el campo de la física teórica, son comparados en esta revisión documental con la percepción de un aparente estancamiento disciplinar, derivado fundamentalmente de la inamovilidad que circunscribe a la Historia al estudio del pasado. La forma de concebir el tiempo, será también el referente utilizado para demostrar la necesidad de romper con los obstáculos que al desarrollo de la ciencia imponen las barreras disciplinares, para finalmente sugerir a los historiadores el tomar en cuenta las potencialidades teóricas y pedagógicas de las bifurcaciones como modelo explicativo alternativo a la representación lineal.
\end{abstract}

Palabras clave: futuros alternos; interdisciplinariedad; tiempo; universos paralelos.

\section{ABSTRACT}

This paper attempts an exercise disciplinary approach between two sciences with different objects of knowledge that coincide in time study as a fundamental coordinate. This notion paradoxically makes the difference between the two, so it will be here addressed as a factor crosscheck between the linear conceptions of it prevails in history versus multidimensionality from which he thinks in physics. The extraordinary advances made in the field of theoretical physics, are compared in this documentary research with the perception of an apparent stagnation discipline, basically stemming from the tenure history circumscribing the study of the past time. The way of conceiving time, will also be the benchmark used to demonstrate the need to break the barriers to the development of science impose disciplinary barriers, finally suggesting to historians taking into account the theoretical and pedagogical potential of bifurcations explanatory model as an alternative to the linear representation.

Keywords: alternate futures; interdisciplinarity; parallel universes; time. 


\section{INTRODUCCIÓN}

Este artículo tiene por objeto de análisis la noción del tiempo desde la visión de la Física y la Historia. El titulo la flecha del tiempo sugiere un fluir en una determinada dirección, generalmente representada en una línea recta que avanza de izquierda a derecha para indicar una trayectoria de recorrido a lo largo de tres etapas: pasado, presente y futuro, la cual es ordenada a discreción para construir una secuencia aparentemente irreversible.

Sobre los supuestos de dirección y secuencia, y en la certeza de la irreversibilidad de lo ocurrido, los historiadores despliegan la explicación de los eventos humanos en el pasado, no obstante en física la realidad se concibe de un modo radicalmente distinto, de allí que en los razonamientos que a continuación se despliegan, se intente un acercamiento disciplinar entre la concepción temporal de la historia y el tiempo de los físicos.

Se trata de una investigación analítica sustentada en una revisión de fuentes documentales, en la cual se expone en un recorrido comparativo los fundamentos epistemológicos de ambas ciencias desde una perspectiva inter y transdiciplinar.

\section{DISCUSIÓN}

Dos perspectivas: una escalar y otra dimensional hacen la diferencia en el modo en que ambas ciencias conciben el tiempo. La física estudia el funcionamiento de un universo inconmensurable y a la vez finito del que forma parte el hombre, en la que el tiempo y el espacio constituyen, desde Einstein lo sabemos, un mismo continuo de cuatro dimensiones, tres espaciales y una temporal donde el pasado, presente y futuro tal como son culturalmente representados no existen. Por su parte la historia estudia el tiempo de la evolución de las civilizaciones humanas en la tierra, de las que forman parte todas las ciencias como productos culturales, en ella se sigue manejando la noción de espacio que se tubo hasta finales del siglo XIX, reducida al sustrato geofísico donde ocurren o transcurren los eventos. La física estudia fenómenos del universo que por eones anteceden al hombre y ante él se rebelan, haciendo que este se vea forzado a interpretarlos en tanto está en juego su misma supervivencia, mientras que la historia es un invento humano que tiene por objeto acopiar, ordenar e interpretar los eventos societales ocurridos en el pasado.

Los saberes que dieron origen a ambas ciencias pueden ubicarse en la prehistoria. De hecho el monumento mesolítico de Aberdeenshire, Escocia, especie de calendario de piedra construido a partir de la observación de las fases del Sol y de la Luna, atestigua la preocupación del hombre por comprender el transcurrir del tiempo a través de la interpretación fenoménica de los astros 8.000 años antes de Cristo. La Historia comienza miles de años después durante la última mitad del IV milenio A. C, con la invención de la escritura y el registro de los eventos en manuscritos de escritura cuneiforme.

Corría la edad del bronce en Sumeria Mesopotámica donde encontramos dos datos importantes que dejan constancia de la antigüedad de la presencia de la historia para entonces como arte, también de la física como campo diferenciado del conocimiento. El primero remite a registros arqueológicos citados por Binkley (1998), que revelan la más antigua evidencia del oficio de historiador; el cual se le atribuye a una mujer, la sacerdotisa Enheduana de Uruk, hija del rey Sargón I de Acadia, la primera persona que registró su autoría en documentos escritos en 
Sumerio cuneiforme que datan del 2.300 A.C.

Como segundo dato, la arqueología también da cuenta de la existencia de documentos babilonios que evidencian la utilización de cálculos que hoy podríamos llamar físicos, destinados a interpretar el movimiento de los astros, véase (Shea 1980). No obstante el comienzo de la Física en su status científico se gestó partir de las leyes que formulara Newton en 1697 en el compendio Principia Mathematica, tratado de teoría general con el que todavía hoy pueden calcularse órbitas de planetas y satélites, trayectorias y velocidades de objetos en el espacio y efectos gravitacionales mutuos entre cuerpos astronómicos, cuyos axiomas alcanzaron el rango de leyes y desde luego constituyen la materialización de un conocimiento científico especializado, construido por acumulación de acuerdo a la taxonomía aportada por Thomas Khum en 1962.

Newton en efecto, construye un modelo explicativo que equipara al universo con un mecanismo de relojería; exacto y predecible. Sin embargo, en las comunidades científicas las verdades son relativas y efímeros los consensos teóricos, por ello la vigencia de estas teorías que en su momento fueron revolucionarias se mantuvo casi por 300 años, hasta que a finales del siglo XIX fueron frontalmente cuestionadas por Henri Poincare, físico y matemático francés quien literalmente despedazó el modelo newtoniano del sistema solar, al demostrar mediante ecuaciones matemáticas que estas leyes solo se ratificaban en el cálculo de interacción gravitacional entre dos cuerpos a velocidad constante, no obstante se derrumban con la entrada en juego de un tercer cuerpo, por lo que en adelante se desató un proceso de contrastación que termino por derrumbar la arquitectura de un universo estable regido por principios invariablemente exactos, para dar paso a nuevas teorías que por el contrario describen un universo entrópico que se desplaza aleatoriamente entre el orden y el caos, algunas de las cuales debelan la existencia de planos ocultos de la realidad por ahora no demostrables experimentalmente, cuya presencia es sin embargo percibida por el modo en que sus efectos inciden en la dinámica del cosmos.

Desde ese portal a la incertidumbre surgen un conjunto de planteamientos que ponen al descubierto la existencia de universos alternos al que habitamos, una realidad no disponible a la percepción sensorial cuyos principios explicativos sustentados en el concepto geométrico, espacio tiempo relatividad de Einstein, tienen su punto de partida en el modelo penta dimensional Kaluza-Klein, propuesto en 1919 por Theodor Kaluza y completado en 1926 por Oskar Klein, del cual han derivado una serie de sorprendentes teorías que dan cuenta de la existencia de universos paralelos al nuestro con los que compartimos el continuo espacio-tiempo, que para los efectos se descubre seccionado en múltiples dimensiones, a las que Feynman citado por Hawking (1994), denomina la suma de historias, que viene a ser el resultado del agregado de todas las historias posibles del universo, es decir de todos los diferentes continuos espacio-tiempo.

Entre estas teorías vale destacar la de los Universos Paralelos o el multiverso, formulada en 1957 por Hugh Everett y más recientemente el principio de súper simetría, que constituyendo una versión acabada del modelo Kaluza- Klein, ha venido a ser una especie de trampolín para el lanzamiento de la denominada revolución de las cuerdas, cuyas ideas iniciales les son atribuidas a Jöel Scherk y John Henry Schwarz en 1974, y sucesivamente desarrolladas hasta lo que hoy se conoce como la revolución de las súper cuerdas propuesta por Edward Witten, 
quien en 1995 formuló la hipótesis de la existencia de 11 dimensiones, es decir once universos paralelos, a cada uno de las cuales denomina P-branas, haciendo alusión a la función separadora en la continuidad, que en la naturaleza caracteriza a las membranas.

Este breve recorrido por la evolución del principio Kaluza - Klein, basado en los aportes de Green (2006), tiene por objeto significar el alto nivel de verosimilitud y de argumentación científica que dentro de los exigentes estándares de la física teórica ostenta la teoría de los universos paralelos, la cual emerge dentro del marco de legitimación surgido a principios del siglo XX con la irrupción de dos grandes modelos explicativos del universo. Se trata de la Teoría general de la relatividad formulada por Albert Einstein en 1905, y la Mecánica Cuántica cuyos cimientos fueron edificados en 1900 por el físico alemán Max Planc.

La Teoría de la relatividad se ocupa de las grandes escalas del universo, por ello se enfoca en la interacción entre masa, gravedad y energía en el cosmos y el macrocosmos, de ella la Teoría de los Universos Paralelos toma el concepto del continuo tiempo-espacio o cronotopo. Por su parte el centro de atención de la mecánica cuántica es la escala microscópica, el mundo subatómico, cuyo comportamiento se rige por leyes distintas a las observadas para el resto del cosmos.

Desde esa perspectiva, uno de los patrones observados, referidos como argumento por la Teoría de los Universos Paralelos es el principio de superposición de estados, el cual en líneas generales indica que un electrón e hipotéticamente cualquier objeto material, puede estar simultáneamente en dos o más locaciones, no obstante una vez observado se materializa en una de ellas, una condición también denominada estado cuán- tico, cuya explicación a todas luces indica que a escala subatómica el mundo no se comporta como lo hace en nuestra escala, en la que un objeto únicamente puede ocupar un solo lugar al mismo tiempo.

La duda en cuanto a la aplicación del principio de superposición de estados en objetos macroscópicos, quedo ingeniosamente despejada en 1935 con el Gato de Shoedringer, experimento mental desarrollado por el físico austriaco Edwin Shoedringer, dirigido a demostrar una serie de hipótesis en torno al comportamiento especifico de las partículas subatómicas en su función de onda. Tras ese objetivo ideó un experimento que consistía en encerrar un gato, en una caja provista de un dispositivo programado con un $50 \%$ de probabilidades de liberar un gas venenoso a partir de la descomposición aleatoria de una partícula radiactiva. Para los efectos, mientras el animal no estuviera a la vista, tendría $50 \%$ de posibilidades de estar vivo y $50 \%$ de posibilidades de estar muerto, y así permanecería en los dos estados, vivo y muerto al mismo tiempo y en el mismo lugar, hasta que el acto de apertura de la caja y la subsiguiente acción observadora, determinara o en este caso sentenciara su condición.

Este experimento ha tenido diversas interpretaciones desde entonces, entre ellas la célebre interpretación de Copenhague y la de muchos mundos o many worlds, que es la que aquí nos interesa, en tanto al sugerir que el gato podría estar vivo y muerto en distintos universos, constituye una aseveración que fractura nuestra certeza en torno a la existencia y el ordenamiento de los seres y las cosas que percibimos, al someter a dura prueba una condición ontológica innata que se reconoce incapaz de pensar de otra manera la ubicación temporal de los eventos.

El mayor aporte para la definición del tiempo fue el de Einstein, en tanto en su 
concepción del continuo temporo-espacial, el tiempo deja de ser la pasiva mesura del trascurrir de los eventos, para transformarse en factor influyente a la vez que influido en y por su ocurrencia. Así lo deja expresado Hawking:

Este fue el mayor triunfo de Einstein. Su descubrimiento transformó por completo nuestro modo de concebir el espacio y el tiempo. Ya no constituían un fondo pasivo en el que sucedía una serie de acontecimientos. Ya no podíamos imaginar el espacio y el tiempo como en un perpetuo transcurso, sin quedar afectados por lo que sucedía en el universo. Muy al contrario, se trataba de unas cantidades dinámicas que influían y eran a su vez influidas por los acontecimientos que allí ocurrían. (Hawking 1994:52)

Hoy seguimos teorizando acerca del tiempo, pero ahora sabemos que no necesariamente se da en línea recta, o por lo menos no se mueve en una sola dirección, en tanto la geometría que se le asigna es solo una representación, cuyo encadenamiento lineal vendría a ser un convencionalismo derivado de la básica premisa Euclidiana que expresa "dos puntos determinan una recta". En este caso pasado, presente y futuro resultan en tres puntos que efectivamente hacen una recta, pero con ellos bien podría construirse un triángulo de no estar alineados, porque la línea recta al fin y al cabo viene a ser solo un conjunto de puntos ordenados para satisfacer la idea de relación entre coordenadas estrechamente asociadas a la representación espacial.

Y como quiera que de puntos hablamos, justo en este me asaltan la memoria las enseñanzas de mi maestra del quinto grado, quien alguna vez en clase de geografía, intentando explicar la redondez de la tierra, y luego de dibujar y borrar el ejemplo del barquito de vela de Aristóteles, hizo que concentráramos la atención en la punta de una tiza que sostenía con la mano derecha extendida hacia nosotros, seguidamente marco una " $X$ " en el centro del pizarrón, asegurándonos que si partiéramos desde esa marca y recorriésemos todo el planeta rayando la trayectoria en línea recta y sin desviarnos, al final arribaríamos directamente a ella por el extremo contrario al punto de partida.

Cuando mi buena maestra Dora, maestra de las de antes, hacia el trazo de tiza desde el punto central del pizarrón hasta el extremo derecho del marco, para luego hacerlo converger al mismo punto desde el extremo izquierdo, luego de dar una vuelta imaginaria alrededor del mundo, que por supuesto incluía ambos extremos de la pared donde colgaba la pizarra, de seguro tenía una idea de la magnitud escalar del universo por su inconmensurabilidad. No obstante era poco probable que conociera las ecuaciones de Friedman que demuestran su curvatura, porque en efecto los objetos lanzados al vacío tienden a desplazarse en línea recta, sin embargo dada la curvatura del continuo, sus trayectorias se inclinan por efectos del campo gravitatorio. Así las cosas, tal como sucede con la forma esférica del planeta que viene a ser una superficie finita sin límites ni bordes, toda línea recta que se prolonga al infinito termina siendo una curva que al final resulta en círculo.

Hablamos entonces de un espacio curvado por efectos de la materia y la energía que contiene, el cual conforma con el tiempo un mismo continúo en la trama de un universo finito, ¿uno entre tantos? Y siendo uno mismo el continuo, ¿Por qué entonces tal como resultaría del completar un ciclo de recorrido en círculo en el espacio, no se podría retornar al punto de partida de un evento, pero en el tiempo?

Se trata de una posibilidad inconcebible que solo tendría cabida en el espacio imaginario de los físicos, para la cual han pensado en el 
horizonte de sucesos, como el momento propicio o el lugar inaprehensible, o "no lugar" donde cualquier cosa puede ocurrir para afectar la continuidad del tramado espacio-tiempo. El horizonte de sucesos como fenómeno astrofísico, vendría entonces a ser un evento impredecible el cual, de acuerdo los teoremas que se atribuye Hawking (1994), formulados conjuntamente con Penrose en 1970, resulta ser la consecuencia del hecho de que el espacio-tiempo se curve sobre sí mismo.

Hasta aquí hemos hablado de rectas y curvas devenidas en representación lineal, que solo es eso, una representación. No obstante la noción de tiempo que como ya se dijo, también es una representación, aunque no necesariamente lineal, tampoco es rígido, dado que en teoría el continuo temporoespacial puede contraerse, expandirse y desdoblarse en múltiples dimensiones o universos paralelos, que bien pudieran ser similares pero con eventos históricos diferentes, totalmente distintos, o bien, idénticos al nuestro.

Entre todas esas opciones me interesa aquí hurgar en la posibilidad de la existencia de un universo paralelo idéntico al nuestro, en tanto allí subyace la posibilidad de viajar hacia atrás en la flecha del tiempo, con una intención expresa, intervenir el pasado para modificar el presente.

Se podría decir que esta pretensión que excita la imaginación en la cultura del siglo XXI, se puso de moda con el éxito alcanzado en el cine por el género ciencia ficción, y no es así, en tanto se trata de una posibilidad que ha estado presente en el imaginario de las civilizaciones que nos anteceden. Totalmente admisible para la mente humana, que por siglos han contemplado la aventura del viaje astral para intervenir el pasado, o bien adelantando la flecha del tiempo, con la expresa intención de conocer el futuro para luego regresar al presente, más que para echar el cuento, para contrarrestar su carga de incertidumbre.

Un antecedente milenario de esta cara aspiración, lo constituye la Epopeya de Gilgamesh, documento mesopotámico escrito según D'Agostino (2007), en Acadio cuneiforme, datado entre los años 1300 y 1000 AC, cuya compilación atribuida al escriba Sin-Liqe-Unninni, constituye un hallazgo de alto valor arqueológico tanto para la historia como para la literatura universal.

Su mención en este ensayo no tendría la misma relevancia, a no ser por el hecho de que en esa épica se detecta por primera vez en la historia, la narración de un viaje realizado por el personaje -Gilgamesh- a través de las barreras del tiempo y el espacio, en busca de la inmortalidad. El viaje en el tiempo viene a ser por lo tanto una de las más antiguas fantasías de la humanidad, hoy convertida en posibilidad remota pero posible, gracias al vuelo concedido al pensamiento por la Mecánica Cuántica.

Volviendo al asunto disciplinar ¿Será la historia una ciencia lo suficientemente abierta a los cambios, como para librarse de los amarres de la linealidad del tiempo, así como de la irreversibilidad que los historiadores le atribuyen a los eventos? La interrogante viene al caso, si se analizan los procesos "legitimados" de evolución y revolución que han experimentado otros campos del conocimiento, especialmente las llamadas ciencias duras y muy particularmente la Física, sin embargo en el campo de la historia, o valga decir en sus nichos académicos, la premisa fundamental se mantiene incólume, la circunscripción al pasado.

En justicia no puede negarse la aparición de importantes cambios que se han producido en cuanto a la diversificación del objeto del 
conocimiento en esta disciplina, que si bien continúa enfocándose en los grandes personajes, en las constantes históricas, en los periodos, en los ciclos etc.; también se permite centrarse en los sucesos y las personas comunes y corrientes. De hecho se puede hacer historia general, historia local, historia de vida; se aborda un hecho considerado importante, pero también se puede hacer historia de la cotidianidad, lo cual constituye un significativo avance, si se contrasta con lo que hasta hace poco fue el objeto del conocimiento primordial en la historiografía tradicional.

Desde el punto de vista metodológico también hay amplitud, se permite ir del determinismo al voluntarismo. Se viene de un proceso de apertura en cuanto al delicado asunto de la legitimidad de las fuentes. La historia oral por ejemplo ha logrado finalmente escalar posiciones de respeto frente a la hegemonía de la historia documental, y aunque los prejuicios y los fetiches metodológicos todavía son endémicos; son muchas las barreras hermenéuticas traspasadas en harás de perfeccionar los métodos de investigación.

Sin embargo, existe una condición inamovi ble que aplica para todas las escuelas historiográficas, presente en la misma génesis de la ciencia, desde Tucidides en la Grecia clásica, pasando por el escepticismo metodológico de Von Ranke y el pragmatismo de Max Weber. Y lo es que, en todas las corrientes, aun en las concepciones de avanzada de Lucien Fevre y March Bloch, se mantienen las nociones pre einstenianas del tiempo, siendo el pasado la única dimensión a considerar, lo cual a mi modo de ver, constituye una especie de cepo ontológico que impide pensar más allá de la disciplina.

\section{El tiempo de los historiadores}

Un acercamiento a la comprensión de esta limitación remite a una premisa fundamental. El tiempo de la historia, es según Marc Bloch (1996:52) "el plasma mismo donde están sumergidos los fenómenos y es como el lugar de su inteligibilidad". No es como en física, el de los eventos naturales, ni se ocupa la historia del estudio de cualquier acontecimiento solo porque hubiera ocurrido en el pasado, pues su objeto del conocimiento son las sociedades humanas.

La historia si bien se circunscribe al pasado, es ciencia de los hombres en el tiempo y en los espacios geográficos, el que le toca vivir como especie, el de la cultura, la organización social y el desarrollo económico. El tiempo histórico no obstante, ser representado en perspectiva lineal aparentemente simple, no deja de ser una representación compleja, si se considera la lógica de sus principios explicativos, entre ellos el globalizador, el teleológico, el de causalidad y el principio de cambio y continuidad, tal como lo expone Betancourt (1993).

En torno a estos cuatro principios se edifica a mi modo de ver la explicación histórica, no obstante, como se trata de la secuencialidad del tiempo, nos detendremos en los últimos dos. Sobre la causalidad dice Bravo:

La causalidad es el instrumento mismo de la certeza, por ella el mundo se hace inteligible y posible de ser dominado. Con el dominio de las redes causales el hombre pudo separarse de la razón divina y crear desde su propio centro, nuevas estructuras de dominio. Poder y certeza encuentran su primera manifestación en la causalidad génesis primera de la inteligibilidad y el sentido. Para Hume "todos nuestros razonamientos acerca de cuestiones de hecho parecen fundarse en la relación de causa y efecto" y para Donald Davidson "La causa es el cemento del universo, el concepto de causa es lo que mantiene unida nuestra imagen del universo". (Bravo 1996:14) 
La relación causa efecto, cuya importancia es suficientemente ilustrada; inflada diría yo por el autor de la cita. Vista de ese modo pudiera ser cuestionada por su perfil determinista, no obstante es definitivamente un parámetro decisivo para el ordenamiento secuencial de los eventos en el tiempo, en tanto sirve de base para el despliegue del cuarto y subsiguiente principio, el de cambio y continuidad, a partir del cual se ordenan los procesos sociales del pasado de acuerdo a su permanencia, en periodos de corta, mediana y larga duración.

Este principio permite dividir el tiempo en segmentos construidos a partir del descubrimiento o de la simple selección del suceso, también orienta la develación de la red causal desde su origen, así como el seguimiento de la continuidad de sus efectos hasta que estos se desdibujan de la línea cronológica. Allí comienza el cambio, justo en el punto de fractura que indica el comienzo de un nuevo periodo.

Se trata por supuesto de una representación del proceso, organizada a discreción por el historiador con el objeto de explicar con alguna precisión algún evento. No obstante desde la física, en cada punto de corte de esa representación lineal, ya en su expresión Diacrónica como Sincrónica, está presente el umbral de la bifurcación y con elle una encrucijada con potenciales accesos a múltiples futuros alternos.

Metodológicamente hablando, la periodización como síntesis explicativa de los principios de causalidad y de cambio y continuidad, reviste sustantiva importancia, en tanto permite delimitar un lapso específico del pasado para estudiarlo con mayor precisión. En esa perspectiva, cada periodo será un eslabón de la secuencia de muchos pasados articulados que dan origen al presente. Visto de ese modo, el pasado engendra al presente y después desaparece, pero ¿solo llega hasta allí su función causal?

Para Heidegger (1999), el pasado viene a ser el presente que ya no lo es más. Un decreto de muerte que pudiera resultar convincente en Filosofía, pero no en Historia para la cual el pasado pervive congelado en la memoria de las fuentes, en las que podría esperar milenios hasta ser develado, mucho menos en Física donde el evento ocurrido no solo queda gravado en el tramado del cosmos, sino que prosigue en múltiples cursos de desarrollo paralelo.

Ahora bien, si se compara con las magnitudes escalares y dimensionales que estudia la física, el objeto de la Historia es modesto pues se circunscribe al estudio del pasado, al contrario de la física no necesita ser predictiva, pues se limita a explicar eventos ya ocurridos, por ello ningún historiador se preocupa por construir leyes sobre la base de la observación de regularidades.

No obstante el conocimiento del pasado, puede en algunos casos orientar la previsión de algún desarrollo posterior de los acontecimientos, y en este punto se centra nuestra crítica. El que se trate del estudio del pasado de la humanidad, por ahora y para todos los efectos "irreversible", no necesariamente debería implicar la circunscripción obligatoria a la causalidad uni lineal, ni se debería ignorar, dado que la historia es una ciencia cuyo objeto del conocimiento es inseparable del tiempo, una teoría que trastoca cualquier aseveración que en adelante se formule en relación a esa noción, como lo es el planteamiento de las bifurcaciones.

\section{El punto de bifurcación}

Las bifurcaciones según Prigogini y Stenger (1994), constituyen el instante vital en el que el curso del tiempo toma un rumbo definitivo, una encrucijada con múltiples ramifica- 
ciones, cada una de las cuales ofrece una opción potencial de futuro, la que una vez abierta definirá en adelante la cadena de causalidad de los eventos. Para los efectos la opción tomada será el detonante que determine el desenlace de los acontecimientos, los que al igual que para los historiadores, también para la mayoría de los físicos son irreversibles.

En cada punto de bifurcación del pasado de nuestro sistema surgió un flujo en el cual existían muchos futuros. A través de la iteración y amplificación del sistema, se escogió un futuro y las demás posibilidades se esfumaron para siempre. Así nuestros puntos de bifurcación constituyen un mapa de la irreversibilidad del tiempo. (Briggs y Peat 1990:49)

El compás de realidades alternas que se abre a partir de este singularísimo evento, la chispa detonante que en ciencias sociales pudiera equiparase al concepto "la coyuntura", no constituye un tema exclusivo de la física cuántica, de hecho en Planificación Estratégica, en salas de análisis situacional por ejemplo, la practica predictiva de futuros alternos a partir de una determinada coyuntura, se maneja asociada a la categoría "escenarios", que suele numerarse en orden probabilístico de ocurrencia en primero, segundo, tercer, hasta el " $\mathrm{N}$ " escenario. No obstante, en estricto sentido disciplinar, la posibilidad de la existencia de cadenas alternas de causalidad no es totalmente ajena a la historiografía. En efecto, en alguna que otra reconstrucción del pasado está implícito el espectro de las bifurcaciones desde el momento en que el autor, más allá de conseguir descifrar la cadena causal del evento, se aventura en la formulación de conjeturas en torno al por que no sucedieron tales o cuales situaciones que pudieron haber ocurrido, o del ¿cómo hubieran sido las cosas, si se hubiera dado tal o cual circunstancia? Y aún más, Fontana (1976) cita la existencia de un enfoque historiográfico que cuestionando la superficialidad de la perspectiva unilineal, profundiza en el análisis de las rutas alternas del curso de la historia, se trata de la historia constelada de Walter Benmjamin, (18921940), notable filósofo de la Escuela de Frankfort.

Y habría que suponer que para entonces, a mediados del siglo pasado, igual que ahora no faltaría quien desde el pudor disciplinar exigiera atenerse a los hechos, tras el argumento de que un evento histórico únicamente puede interpretarse si y solo si ya ocurrió, de modo que las hipótesis tejidas en torno a lo que pudiera haber sucedido, no pasarían de ser vulgares especulaciones, o elucubraciones folclóricas dignas de magos y adivinos. Como ejemplo tómese nota de esta afirmación de Pages, citada por Valera:

... el historiador sólo puede interpretar la necesidad de un hecho histórico en la medida en que ya haya sucedido, pues todas las hipótesis que barajase sobre lo que hubiese podido conocer si se hubiese actuado de otra manera constituyen, en el mejor de los casos, meras especulaciones. (Valera 2001:5)

Volviendo al asunto de la irreversibilidad del tiempo. Si se le analiza con detenimiento, el rotundo escepticismo con el cual se le considera está anclado en un sólido asidero, dado que la posibilidad de encontrar aplicaciones prácticas a la teoría de los Universos Paralelos, que pudieran transferirse desde la Física a la Historia, pasa por la solución de dos problemas; uno de orden empírico y otro de orden teórico.

El problema empírico tiene que ver con la tecnología en tanto todavía no se vislumbra la posibilidad de construir dispositivos que permitan acometer semejante reto, me refiero al viaje en el tiempo. En cuanto a lo teórico, por el lado de la física, el problema está 
en la limitación que impone el concepto de irreversibilidad, por el lado de la historia, su inamovible radicación en una única dimensión temporal, dado que desde siempre la historia ha sido y lo seguirá siendo hasta nuevo aviso, el estudio del pasado.

La posibilidad de viajar en el tiempo es tan remota, que aun en el campo, "abierto a cualquier idea", de la física, ha sido capaz de granjearse prestigiosos detractores, Hawking (1994:106), por ejemplo, afirma que: "la prueba mejor con que contamos acerca de la imposibilidad actual y perenne del viaje por el tiempo es que no hemos sido invadidos por hordas de turistas del futuro". Un argumento imbatible con el que casi todos podemos estar de acuerdo. Sin embargo la reversibilidad del tiempo, aunque infinitamente improbable, es para autores como Ilya Prigogini, una posibilidad cierta, argumentando que en la naturaleza nada esta negado y no todas las leyes de la física se han descubierto, empero la magnitud del impedimento es enorme si se considera que el punto de partida es en sí mismo una negación, la de concebir el tiempo de otro modo dentro o fuera de la racionalidad científica, por cuanto justamente la noción limitada que en torno a él se ha construido es el obstáculo, un impedimento que por estar basado en una imposibilidad, la de pensar, pudiera ubicarse en la categoría del obstáculo epistemológico.

El Obstáculo Epistemológico se hace sentir, cuando el intento de encausar la verosimilitud de una teoría se atasca en una situación que podría llamarse de "juego trancado", dada la dificultad que por ahora tienen los físicos en demostrar la posibilidad de revertir el tiempo, con el agravante de que para la ciencia de la historia se trata de una premisa negada, en tanto no forma parte del interés de quienes se limitan a trabajar con hechos ya ocurridos, desde la plena certeza de que estos no pueden modificarse.

\section{Del obstáculo de la naturaleza, a la natu- raleza del obstáculo}

Se le atribuye al filósofo y ensayista Francés Gastón Bachelard (1884-1962), la mención inicial de una concepción que estima que la ciencia progresa en la medida en que supera los obstáculos epistemológicos que dificultan su avance, al considerar que en el proceso de producción del conocimiento, se conoce "en contra del conocimiento anterior, destruyendo conocimientos mal adquiridos o superando aquello que, en el espíritu mismo, obstaculiza la espiritualización”.

Por ello Bachelard (2004:42), define como Obstáculos Epistemológicos, a todos aquellos entorpecimientos y confusiones que se experimentan durante el acto de conocer, y en ese sentido expresa que:
Hay que plantear el problema del conoci- miento científico en términos de obstácu- lo, que no son obstáculos externos, pues es en el acto mismo de conocer, íntima- mente, donde aparecen, por una especie de necesidad funcional, los entorpeci- mientos y las confusiones... causas de estancamiento y hasta de retroceso, causas de inercia que llamaremos obstá- culos epistemológicos. Es necesario romper estas barreras para poder conocer en contra de un conocimiento anterior, destruyendo conocimientos mal adquiri- dos o superando aquello que, en el espíri- tu mismo, obstaculiza a la espiritualiza- ción. (Bachelard 2004:26)

En este caso, la barrera viene a ser nuestra noción del tiempo, que hoy por hoy representa un obstáculo equiparable al que para la navegación llegó a representar hasta un pasado reciente, cuando no se disponía de la tecnología que en la actualidad nos asiste, la idea de la inconmensurabilidad del mar. Por ello, en los albores del siglo XIX, la colosal extensión del mar como barrera natural, dificultaba la aceptación de una teoría que surgida como alternativa a la del puente de Bering, atribuía el poblamiento americano a 
una corriente migratoria multirracial, integrada por pueblos de origen australiano, malayos, melanesios y polinésicos, que a lo largo de un periodo de 14.000 años cruzaron en balsas el océano pacífico para asentarse en las costas de América del sur. En efecto, la fragilidad de las embarcaciones, la inestabilidad atmosférica durante la travesía, la inexistencia de instrumentos de navegación y por encima de todo, la enorme distancia expresada en los miles de kilómetros que separan cualquier isla del Triángulo Polinésico con respecto a las costas chilenas y peruanas, se constituyeron en elementos suficientes para negar posibilidades a esta teoría; contrastada desde la perspectiva del mar como obstáculo.

Fue solo cuando empezó a considerarse la perspectiva del mar como ruta, que los conocimientos aportados por la meteorología y la hidrodinámica aplicados a la hipótesis de la contribución del viento y las corrientes marinas para dar rumbo y propulsión a las balsas, sumado a esto los aportes de la Arqueología y la Biología molecular en cuanto al hallazgo de vocablos, utensilios similares y coincidencias fenotípicas entre los pueblos a ambos extremos del océano y sobre todo, cuando empezó a considerarse como variable, la persistente tenacidad del hombre, que la hipótesis migratoria del pacifico se legitimó hasta ser considerada parte de la gran teoría general del poblamiento múltiple de América ,cuyo máximo exponente es el etnólogo francés Paul Rivet (1979).

Como se ve, el asunto de la superación de obstáculos epistemológicos es una cuestión de perspectiva, que pasa por el manejo de un pensamiento complejo abierto a todas las posibilidades y por lo tanto, capaz de sortear los límites que imponen las disciplinas.

\section{La barrera tecnológica}

El problema empírico como impedimento a la legitimación de esta teoría ciertamente radica, en las limitaciones tecnológicas. $\mathrm{La}$ máquina del tiempo que en las películas nos soluciona el problema taladrando a discreción agujeros de gusano en la trama del continuo, tal cual lo haría el Delorean, está muy lejos de construirse.

Ni que hablar de la posibilidad de ralentizar el tiempo a bordo de un vehículo capaz de desplazarse a una velocidad igual o cercana a la de la luz, una proeza imposible en tanto viola las leyes de la física. No obstante la historia de la sinergia entre ciencia y tecnología, desde sus inicios indica que la clave de apertura al campo infinito de las posibilidades está en el vuelo ilimitado de la imaginación, un vuelo que por ahora solo tiene en el cálculo físico matemático una escala de supervivencia en la frontera que separa lo posible de lo imposible, tal como hace milenios lo seria para los navegantes Polinesios la Isla de Pascua en medio de la nada.

También indica la historia, que la complementariedad de esta relación hace que la ciencia genere tecnología, y por su vez la tecnología ratifique y actualice las teorías científicas. En ocasiones la claridad teórica induce la construcción del dispositivo tecnológico, por ejemplo, el conocimiento del patrón orbital del átomo condujo al desarrollo de la energía nuclear, o el de la fisiología del aparato renal condujo a la construcción de la máquina de diálisis, y en sentido contrario, la tecnología de procedencia empírica, es decir la que viene con la experiencia, vía ensayo y error, confirma y actualiza a veces sin intención las premisas de una teoría, por ello la invención de la Brújula y el Sextante, así como las mejoras sustantivas en las construcciones navales, determinaron la confirmación de las hipótesis de Aristóteles en cuanto a la redondez de la tierra, y consecuencialmente condujeron al descubrimiento "por parte de los europeos" de un nuevo continente en el siglo XIV. 
Volviendo al problema, la dificultad que entraña la noción que tenemos del tiempo se crece en la medida en que su transcurrir condiciona la percepción de la secuencia y la velocidad de los procesos en el intento de medirlos de acuerdo a su duración. De hecho la percepción de la extensión de los periodos, está a su vez condicionada por la ubicación del sujeto cognoscente en la línea del tiempo, por ello vistos del presente al pasado los procesos nos parecen cortos. En cambio cuando se trata de vislumbrar las proyecciones futuras de un proceso desde el presente, el peso de la incertidumbre y la carga subjetiva hacen que los periodos parezcan largos. Sin embargo la historia guarda en el viejo adagio presentista, "lo que ayer parecía imposible hoy es realidad", la más esperanzadora de todas las premisas.

No en vano la ambición milenaria del hombre por imitar el vuelo de los pájaros, anhelo plasmado a la posteridad en la fábula griega de Ícaro, se vio por fin satisfecha en 1903 cuando los hermanos Wright hicieron volar el primer avión, y desde de allí no se hizo esperar el gran salto tecnológico que para la humanidad significo el desembarco del hombre en la luna en 1969. Todo es cuestión de tiempo.

Solo que aquí el tiempo es el problema, su condición irreversible el obstáculo. Un obstáculo cuya superación, considerada en perspectiva del presente al futuro parece imposible. No obstante tal como ocurrió con las teorías de Ptolomeo y Copérnico es solo "cuestión de tiempo" para que tengamos la tecnología, la misma que con su avance potencia la comprensión de lo desconocido, porque la ciencia funciona así, forzando barreras.

De hecho, medidos hoy en la era de los drones, desde el avión de los hermanos Wright al alunizaje solo transcurrieron 63 años. Se estima que en los próximos 50 años plantaremos nuestra huella en Marte, y todo parece indicar que estamos al borde de un gigantesco salto tecnológico basado en el descubrimiento de fuentes poderosísimas inagotables y maleables de energía, como lo son el aprovechamiento absoluto de las potencialidades del hidrogeno y el desciframiento del patrón estructural de la antimateria.

Finalmente y haciendo uso de la claridad que otorga la percepción de una realidad compleja, me ubico en el criterio de que esta, pese a ser una sola tiene múltiples niveles, todos con objetos del conocimiento tan específicos que ameritan ser focalizados por miradas especializadas, cuya profundidad pudiera incrementarse exponencialmente con la complementación de otras miradas disciplinares en la interdisciplinaridad, y más allá con la superación de barreras disciplinares desde la transdisciplinariedad.

En cuanto a la relación que en este artículo se ha querido forzar entre dos ciencias que ciertamente no son afines, pero que coinciden en la primacía que ambas le otorgan al tiempo, puede decirse que como ciencias se constituyen en los más refinados instrumentos de interpretación de la realidad, los cuales de manera explícita en la física e implícita para la historia, fundamentan sus explicaciones en la captación de regularidades, y al igual que todas las otras ciencias, más allá del carácter explicativo, en ambas se perfila una pretensión predictiva que tiene por objeto el control de los eventos.

Una intención manifiesta que abiertamente inspira el discurso de los físicos, que sin embargo intenta pasar desapercibida en el discurso historiográfico que con uñas de cristal se aferra al pasado, en un intento frustrado, porque en él se cuelan posiciones muy bien argumentadas que dejan ver que la luz 
que alumbra la salida dentro túnel del tiempo proviene del futuro, entre ellas tómese nota de ésta publicación de Vargas (1999), cuyo título se explica por si solo: La historia como futuro, o afirmaciones de este tenor, expresadas en la siguiente cita:

La historia es una ciencia que sin sacrificar la rigurosidad de la aplicación del método científico para la investigación, tiene ontológicamente la necesidad de contribuir al desarrollo y a la felicidad de los hombres en sociedad y en este sentido la historia es la ciencia del tiempo: pasado, presente y también futuro. Es decir que su razón de ser la encontramos no en el conocimiento erudito, si no en la aplicación del conocimiento histórico del pasado y del presente en el presente mismo, para incidir intencionalmente en el devenir de los pueblos. (Paez 2002:21)

Como se ve, no solo se trata de que la huella hacia el futuro o al pasado según sea el caso, se presente inseparable de las otras dos estaciones de la representación del tiempo, si no de la posibilidad de controlar los eventos haciendo uso del poder que pudiera otorgar el conocimiento anticipado del curso que pudieran tomar los acontecimientos, desde el punto singularísimo de la bifurcación.

Una posibilidad que pese a ser remota demanda para su viabilidad del concurso de mentalidades abiertas que faciliten "el salto a la complejidad", una condición que (Morin 1994:19) describe como "el tejido de eventos desordenados e inextricables que constituyen nuestro universo fenoménico", solo que el desorden es algo que de entrada resulta incómodo para los historiadores, quienes por el contrario dedican sus mejores esfuerzos a ordenar los eventos en prolijas líneas causales, a quienes además les correspondería deslindarse del prejuicio levantado durante el siglo pasado en las ciencias sociales como conjuro contra el positivismo, que ordenaba no intentar parecerse y mucho menos utilizar métodos propios de las ciencias naturales.
Una sugerencia inútil, si se considera que el escepticismo positivista dejo para siempre su aporte benefactor como referente fundamental en cuanto a la diferenciación del conocimiento científico con respecto a otros tipos de conocimientos, pero más allá de eso, y pese a que como modelo normativo se conserve cual fetiche en algunas comunidades científicas, este ya no es capaz de imponer regla alguna a la producción de conocimientos, por lo que de lo que se trata ahora es de superar los obstáculos que entorpecen el desarrollo de la ciencia en general y de la Historia en particular, en cuyo ámbito la preocupación por el predominio de tendencias ortodoxas demarcadoras de impermeables fronteras disciplinares, no es un asunto nuevo que pueda ser cuestionado hoy desde el pensamiento complejo.

De hecho se trata de una problemática prevista en su época por March Bloch (1886-1944), al percibir el secuestro de teorías y métodos por parte de quienes se hubieran erigido cual sumos sacerdotes en defensa del oficio. De allí la siguiente cita, inspirada en un pasaje de la mitología griega en el que una deidad advierte a su aprendiz contra las tentaciones del cambio.

"...este tema o esta manera de tratarlo", dice el guardián de los dioses términos, "es lo que probablemente puede seducir. Pero ten cuidado, ;oh efebo!: eso no es historia". ¿Acaso somos una juraduría de los tiempos antiguos para codificar las tareas permitidas a las gentes de oficio y, una vez cerrada la lista, reservar el ejercicio a nuestros maestros con patente? Los físicos y los químicos son más sabios; hasta donde yo sé, jamás se les ha visto pelear por los derechos respectivos de la física, de la química, de la química-física o - suponiendo que este término existade la física-química. (Bloch 1996:54)

No es casual que el autor al criticar la acción retrógrada de las cofradías en el seno de la 
comunidad historiográfica, lo hiciera en comparación con las ciencias naturales, incluso y como quiera que el texto también denota una defensa implícita de la interdisciplinaridad, viene al caso resaltar que en algún lugar del tratado "Apología para la Historia", Bloch afirma que la física no hubiera llegado tan lejos de haberse ceñido a las ataduras disciplinares, y justo de eso se trata, de llegar tan lejos como se pueda fracturando ortodoxias y franqueando barreras disciplinares.

\section{CONCLUSIONES}

Aun cuando en estas líneas se ha reiterado la necesidad de ampliar la estructura teórica y por consiguiente los métodos de investigación a través de la práctica interdiscplinar y transdisciplinar, de ningún modo se ha querido sugerir aquí el abandono o el desplazamiento del objeto del conocimiento de la Historia, pues si esta deja de ser el estudio del pasado, dejaría de ser Historia para ser cualquier otra cosa. Una cuestión axiomática que encuentra algún nivel de comprensión entre los cosmólogos, por ello me permito concluir estas reflexiones citando de nuevo a Hawking (1994:106) quien refiriéndose a la posibilidad tantas veces tratada en este trabajo, se permite a bromear, aludiendo las conocidas actitudes de defensa del oficio que suelen esgrimir algunas cofradías historiográficas, con esta afirmación: "parece que las leyes de la física no permiten semejante viaje por el tiempo. Quizá exista un Instituto de Protección de la Cronología que, impidiendo ir al pasado, garantiza la seguridad de los historiadores".

En todo caso, con este artículo solo se ha pretendido tender un cable de comunicación con la intención de forzar alguna brecha en la sólida barrera disciplinar que circunscribe a esta ciencia al estudio del pasado, mostrando a la vez el amplísimo espectro de posibili- dades que para el oficio de historiador y muy especialmente para la enseñanza de esa disciplina, significaría la simple actualización de la noción cronológica, sumada al extraordinario impulso holístico que como teoría explicativa de las redes de causalidad en sus potenciales aplicaciones didácticas, implicaría la consideración epistémica de las bifurcaciones como principio constructivo que entrelaza la trama del tiempo histórico.

\section{REFERENCIAS \\ BIBLIOGRÁFICAS}

Bachelard, G. (2004). La formación del espíritu científico. México DF, México: Siglo XXI editores.

Betancourt, D. (1993). Enseñanza de la Historia a tres niveles. Bogotá, Colombia: Editorial Magisterio.

Binkley, L. (1998). Biografia de Enheduanna, sacerdotisa de Inanna. Pensilvania, Estados Unidos: Universidad de Pensilvania.

Bloch, M. (1996). Apología para la historia o el oficio de historiador. México DF, México: Fondo de cultura económica.

Bravo, V. (julio de 1996). El fracaso y la ceguera de existir. Últimas noticias, 8-9.

Briggs, J. \& Peat D. (1990). Espejo y reflejo, del caos al orden. Barcelona, España: Gedisa editorial. 


\section{Unach}

D'Agostino, F. (2007). Gilgamesh o la conquista de la inmortalidad. Madrid, España: Editorial Trotta.

Fontana, L. (1976). Ascenso y decadencia de la escuela de los Annales. Madrid, España: Akal Editor.

Green, B. (2006). El Universo Elegante. Barcelona, España: Crítica Editorial.

Heidegger, M. (1999). El Concepto de Tiempo. Madrid, España: Editorial Trotta.

Hawking, S. (1994). Agujeros Negros y Pequeños Universos y otros ensayos. Madrid, España: Editorial Plaza \& Janes.

Kuhn, T. (1962). La estructura de las Revoluciones Científicas. México DF, México: Fondo de Cultura Económica.

Morin, E. (1994). Introducción al pensamiento complejo. Barcelona, España: Editorial Gedisa.

Paez, G. (2002). Historia Regional. Investigación y Enseñanza. Caracas, Venezuela:FEDUPEL

Prigogini, I. \& Stenger, E. (1994). Entre el tiempo y la eternidad. Madrid, España: Alianza Editorial

Rivet, P. (1979). Los orígenes del hombre americano. México DF, México: Fondo de cultura económica.

Shea, W. (1980). The Calendars of Ebla. Michigan, Estados Unidos: Andrews University Seminary Studies.

Valera, G. (2001). La Explicación de los Fenómenos Sociales. Revista Fermentum, 11(30), 36-64.

Vargas, I. (1999). La historia como futuro. Caracas, Venezuela: Fondo editorial Tropykos.
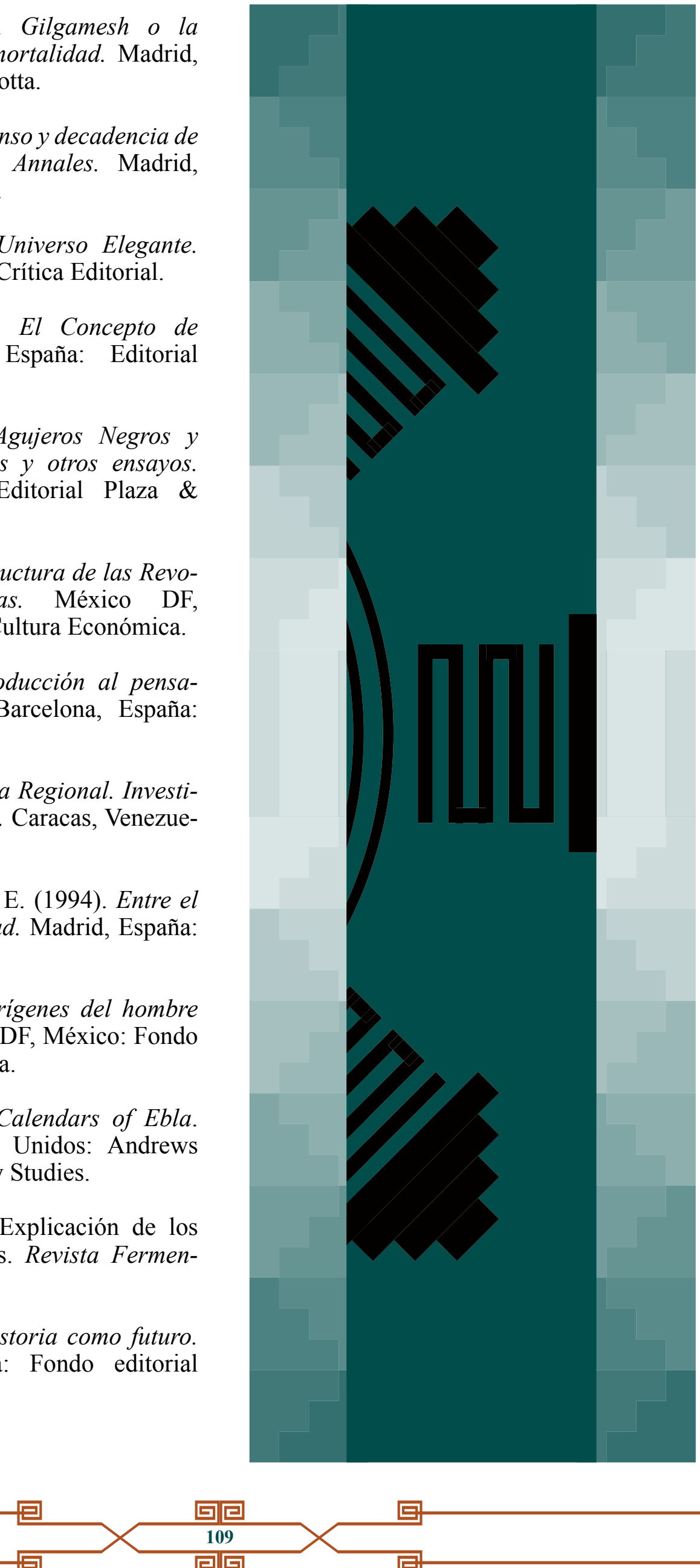